University of Wollongong

Research Online

Faculty of Engineering and Information

Faculty of Engineering and Information

Sciences - Papers: Part A

Sciences

$1-1-2014$

\title{
Topologically guaranteed enhancement of nonlinear optical conductivity of graphene in the presence of spin-orbit coupling
}

Zheng Liu

Shanghai Institute of Microsystem and Information Technology

Matthew Sanderson

University of Wollongong, ms919@uowmail.edu.au

J C. Cao

Shanghai Institute of Microsystem and Information Technology

C Zhang

University of Wollongong, czhang@uow.edu.au

Follow this and additional works at: https://ro.uow.edu.au/eispapers

Part of the Engineering Commons, and the Science and Technology Studies Commons

Research Online is the open access institutional repository for the University of Wollongong. For further information contact the UOW Library: research-pubs@uow.edu.au 


\title{
Topologically guaranteed enhancement of nonlinear optical conductivity of graphene in the presence of spin-orbit coupling
}

\author{
Abstract \\ We demonstrate that a topological invariant term in graphene (e.g., spin-orbit coupling or interlayer \\ coupling induced trigonal warping) can lead to a strong nonlinear optical response. We present a full \\ quantum-mechanical analysis of the third-order nonlinear optical conductivity of the monolayer graphene \\ with a finite Rashba spin-orbit coupling. For energy of the incident photon below the maximum height of \\ the trigonal warping and the Rashba coupling strength exceeding a critical value the nonlinear \\ conductance is enhanced dramatically in comparison with that in the absence of the topological term. \\ Due to the trigonal warping effect and the topologically inequivalent change of the geometry of the energy \\ band, the strong nonlinear effect persists, as long as the incident photon energy is limited below the \\ energy maximum defined by the spin-orbit coupling.

\section{Disciplines} \\ Engineering | Science and Technology Studies

\section{Publication Details} \\ Liu, Z., Sanderson, M., Cao, J. \& Zhang, C. (2014). Topologically guaranteed enhancement of nonlinear \\ optical conductivity of graphene in the presence of spin-orbit coupling. Physical Review B - Condensed \\ Matter and Materials Physics, 90 (23), 235430-1-235430-6.
}

This journal article is available at Research Online: https://ro.uow.edu.au/eispapers/3830 


\title{
Topologically guaranteed enhancement of nonlinear optical conductivity of graphene in the presence of spin-orbit coupling
}

\author{
Zheng Liu, ${ }^{1}$ Matthew Sanderson, ${ }^{2}$ J. C. Cao, ${ }^{1, *}$ and C. Zhang ${ }^{2, \dagger}$ \\ ${ }^{1}$ Key Laboratory of Terahertz Solid-State Technology, Shanghai Institute of Microsystem and Information Technology, \\ Chinese Academy of Sciences, 865 Changning Road, Shanghai 200050, China \\ ${ }^{2}$ School of Physics, University of Wollongong, New South Wales 2522, Australia \\ (Received 1 September 2014; revised manuscript received 27 November 2014; published 22 December 2014)
}

\begin{abstract}
We demonstrate that a topological invariant term in graphene (e.g., spin-orbit coupling or interlayer coupling induced trigonal warping) can lead to a strong nonlinear optical response. We present a full quantum-mechanical analysis of the third-order nonlinear optical conductivity of the monolayer graphene with a finite Rashba spin-orbit coupling. For energy of the incident photon below the maximum height of the trigonal warping and the Rashba coupling strength exceeding a critical value the nonlinear conductance is enhanced dramatically in comparison with that in the absence of the topological term. Due to the trigonal warping effect and the topologically inequivalent change of the geometry of the energy band, the strong nonlinear effect persists, as long as the incident photon energy is limited below the energy maximum defined by the spin-orbit coupling.
\end{abstract}

DOI: 10.1103/PhysRevB.90.235430

PACS number(s): 73.63.Bd, 78.66.Tr, 78.67.-n

\section{INTRODUCTION}

Graphene is a two-dimensional honeycomb lattice of carbon atoms that has been intensely studied because of experimental progress [1-3] and many new physical features such as the half-integer quantum hall effect [4], finite conductivity at zero charge-carrier concentration [5], perfect quantum tunneling effect [6], and ultrahigh carrier mobility [7]. The low-energy electrons in graphene offer an ideal system for studying the relativistic dynamics. A finite spin-orbit coupling (SOC) or interlayer hopping in a bilayer graphene (BLG) offers a mechanism to generate topological change of the low-energy band structure, known as the trigonal warping [8-10].

The spin-orbit coupling in graphene can be intrinsic or extrinsic. They affect the electronic properties of graphene in different ways. The intrinsic SOC [11] leads to a finite gap between the conduction and valence bands. Its modulation may turn a single layer graphene into a topological insulator [12]. The presence of extrinsic SOC results in a $k$-dependent energy shift and deforms the band structure [13]. The effect is manifested through the anisotropic spin splitting of the bands at the $K\left(K^{\prime}\right)$ points [9]. The extrinsic SOC can be regarded as Rashba SOC (RSOC), which is generated by the structure inversion asymmetry of the graphene sheet and has an obvious influence on the linear optical conductivity $[14,15]$. It can be further tuned with an external electric gate voltage, or electrostatic interaction with the substrate. The effects of intrinsic and extrinsic SOCs can be analyzed by using a tight-binding model for monolayer, bilayer, and multilayer graphene [16-18]. The role played by intrinsic and extrinsic SOI in a given physical property is quite different due to three orders of magnitude difference in their relevant energy scales. The energy of the intrinsic SOC [19] is a few $\mu e V$ while that of the extrinsic SOC is in the order of 1-10 meV [20]. A Rashba splitting of $225 \mathrm{meV}$ has been observed in an epitaxial graphene layer on a $\mathrm{Ni}(111)$ surface [13] due to the $\mathrm{SU}(2)$ spin symmetry breaking. Under the RSOC, the low-energy bands undergo trigonal warping deformation at the $K$ and $K^{\prime}$ points of graphene's Brillouin zone (BZ) in the reciprocal lattice. One Dirac point is now split into four gapless points $[9,17,21]$. One of them remains at the $K\left(K^{\prime}\right)$ point and the other three link up an equilateral triangle around the $K\left(K^{\prime}\right)$ point. The warping changes the topology of the low-energy bands at the $K\left(K^{\prime}\right)$ points of graphene's BZ [22,23]. The deformation of their low-energy conical approximation is very similar to what happens in bilayer graphene [24-27]. Mathematically it can be shown that a single layer graphene with SOC and a BLG can be described by an equivalent Hamiltonian.

Graphene has superior optical properties; chief among them is the universal conductance $\sigma_{0}=4 e^{2} / \pi h$ in the lowto visible-frequency regime [28]. Other interesting properties include the dynamic conductivity [29-32], enhanced optical conductivity of bilayer graphene nanoribbons [33] and strong terahertz conductance under a magnetic field [34]. Both SOC and the interlayer hopping change graphene's universal conductance from $\sigma_{0}$ to $6 \sigma_{0}$. This six times enhancement is independent of the strength of SOC or the interlayer hopping, confirming the topological nature of SOC and interlayer hopping [10,23,35]. Graphene is also a strong natural nonlinear material [36-39]. An important question that remains to be answered is whether a SOC changes the nonlinear response in a way that is topologically guaranteed. In this work we shall show that there exists a topologically guaranteed enhancement of the nonlinear optical conductance by SOC in graphene.

\section{MODEL AND NONLINEAR CONDUCTIVITY}

For the monolayer graphene with RSOC the Hamiltonian is given as $\hat{H}=\hat{H}_{0}+\hat{H}_{\text {RSO }}[11,40]$, where $H_{0}$ is the Hamiltonian of a graphene without RSOC and $H_{\mathrm{RSO}}$ is the Hamiltonian for spin-obit interaction. In the tight-binding approximation,

$$
\hat{H}_{0}=\sum_{\vec{k} \in B Z}\left(\hat{C}_{\vec{k}_{A}}^{\dagger}, \hat{C}_{\vec{k}_{B}}^{\dagger}\right) t_{h}\left(\begin{array}{cc}
0 & h(\vec{k}) \\
h^{*}(\vec{k}) & 0
\end{array}\right)\left(\begin{array}{l}
\hat{C}_{\vec{k}_{A}} \\
\hat{C}_{\vec{k}_{B}}
\end{array}\right),
$$

\footnotetext{
jccao@mail.sim.ac.cn

†czhang@uow.edu.au
} 
where

$$
\begin{aligned}
& \hat{C}_{\vec{k}_{v}}^{\dagger}=\frac{1}{\sqrt{N}} \sum_{\vec{k} \in B Z} e^{i \vec{k} \cdot \vec{l}_{v}} \hat{C}_{\vec{l}_{v}}^{\dagger}, \\
& \hat{C}_{\vec{k}_{v}}=\frac{1}{\sqrt{N}} \sum_{\vec{k} \in B Z} e^{-i \vec{k} \cdot \vec{l}_{v}} \hat{C}_{\vec{l}_{v}} .
\end{aligned}
$$

Here $t_{h}$ is the hopping energy $(\approx 2.7 \mathrm{eV}), v=A, B, \hat{C}_{\vec{k}_{v}}^{\dagger}$ and $\hat{C}_{\vec{k}_{v}}$ are creation and annihilation operators for an electron with lattice vector $l$ in sublattice $v . \vec{l}_{B}=\vec{l}_{A}+\vec{\delta}, h(\vec{k})=$ $\sum_{\vec{\delta}} e^{i \vec{k} \cdot \vec{\delta}}$ and the operator $\hat{C}_{\vec{k}_{v}}^{\dagger}$ is given as $\left\langle\vec{r}\left|\hat{C}_{\vec{k}_{v}}^{\dagger}\right| 0\right\rangle \equiv\left|\varphi_{\vec{k}_{v}}(\vec{r})\right\rangle=$ $\frac{1}{\sqrt{N}} \sum_{\vec{l}_{v} \in T_{v}} e^{i \vec{k} \cdot \vec{l}_{v}} \phi_{2 p_{z}}\left(\vec{r}-\vec{l}_{v}\right)$ with $\phi_{2 p_{z}}(\vec{r})$ usually chosen as the $2 p_{z}$ orbit wave function of the carbon atom. For a fixed Bloch wave vector $\vec{k}$, the Hamiltonian $H_{0}$ in the two-dimensional subspace $\mathscr{V}_{k}^{2 D}$ spanned by the basis functions $\left\{\left|\varphi_{\vec{k}_{A}}\right\rangle,\left|\varphi_{\vec{k}_{B}}\right\rangle\right\}$ is

$$
H_{0}^{2 D}(\vec{k})=t_{h}\left(\begin{array}{cc}
0 & h(\vec{k}) \\
h^{*}(\vec{k}) & 0
\end{array}\right) .
$$

When the degree of freedom of the spin is taken into account, the subspace becomes four dimensional with four base vectors,

$$
\begin{aligned}
& \left\{\left|\varphi_{\vec{k}_{A}}\right\rangle \otimes|\uparrow\rangle,\left|\varphi_{\vec{k}_{B}}\right\rangle \otimes|\uparrow\rangle,\left|\varphi_{\vec{k}_{A}}\right\rangle \otimes|\downarrow\rangle,\left|\varphi_{\vec{k}_{B}}\right\rangle \otimes|\downarrow\rangle\right\} \\
& \quad \equiv|\vec{k}, \mu\rangle \in \mathscr{V}_{k}^{4 D},
\end{aligned}
$$

$\mu=1,2,3,4$. The Hamiltonian $H_{0}$ in Hilbert subspace $\mathscr{V}_{k}^{4 D}$ with no spin-orbit interaction is the direct product $H_{0}^{4 D}=$ $H_{0}=\hat{I}_{s} \otimes H_{0}^{2 D}$, where $\hat{I}_{s}$ is the unit operator (matrix) in the spin space.

The RSOC term can be wriiten as $[21,41]$

$$
\hat{H}_{\mathrm{RSO}}=i \sum_{\vec{l} \in T} \sum_{\vec{\delta}}\left[\left(\vec{u}_{\vec{\delta}} \cdot \overrightarrow{\hat{\sigma}}\right) \otimes \hat{C}_{\vec{l}}^{\dagger} \hat{C}_{\vec{l}+\vec{\delta}}-\left(\vec{u}_{\vec{\delta}} \cdot \overrightarrow{\hat{\sigma}}\right) \otimes \hat{C}_{\vec{l}+\vec{\delta}}^{\dagger} \hat{C}_{\vec{l}}\right],
$$

where $\vec{u}_{\vec{\delta}}=-\frac{\lambda_{R}}{d_{0}} \hat{z} \times \vec{\delta}=-\frac{\lambda_{R}}{d_{0}}\left\{-\vec{\delta}_{y}, \vec{\delta}_{x}, 0\right\}, \vec{u}_{\vec{\delta}} \cdot \overrightarrow{\hat{\sigma}}=\frac{\lambda_{R}}{d_{0}}\left(\vec{\delta}_{y} \hat{\sigma}_{x}-\right.$ $\left.\vec{\delta}_{x} \hat{\sigma}_{y}\right), d_{0}=\frac{a}{\sqrt{3}}$. Substituting Eq. (2) into Eq. (4) and after a similar procedure it is obtained that

$$
\begin{aligned}
H_{\mathrm{RSO}}= & i \hat{\sigma}_{x} \otimes\left(\begin{array}{cc}
0 & h_{y}^{+}(\vec{k}) \\
-h_{y}^{-(\vec{k})} & 0
\end{array}\right) \\
& -i \hat{\sigma}_{y} \otimes\left(\begin{array}{cc}
0 & h_{x}^{+}(\vec{k}) \\
-h_{x}^{-}(\vec{k}) & 0
\end{array}\right),
\end{aligned}
$$

where $h_{n}^{ \pm}=\frac{\lambda_{R}}{d_{0}} \sum_{\vec{\delta}} e^{ \pm i \vec{k} \cdot \vec{\delta}} \vec{\delta}_{n}(n=x, y)$. The total Hamiltonian in the subspace $\mathscr{V}_{k}^{4 D}$ is

$$
\begin{aligned}
\hat{H}^{4 D} & =\hat{H}_{0}^{4 D}+\hat{H}_{\mathrm{RSO}} \\
& =t_{h}\left(\begin{array}{cccc}
0 & \varphi_{0} & 0 & i \varphi_{+} \Delta \\
\varphi_{0}^{*} & 0 & -i \Delta \varphi_{-}^{*} & 0 \\
0 & i \Delta \varphi_{-} & 0 & \varphi_{0} \\
-i \varphi_{+}^{*} \Delta & 0 & \varphi_{0} & 0
\end{array}\right),
\end{aligned}
$$

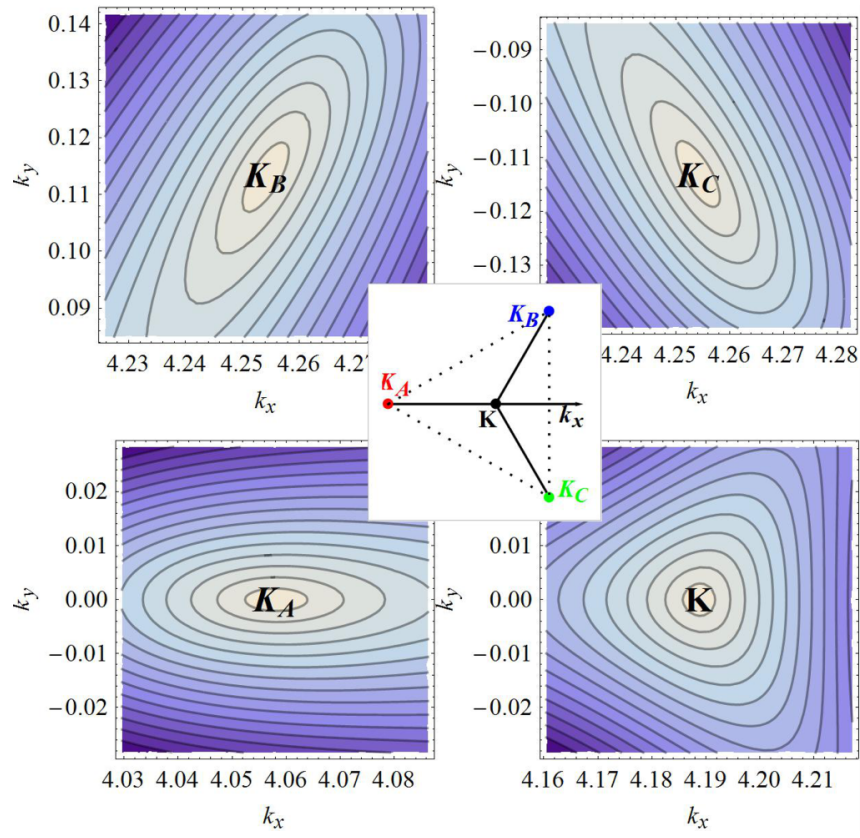

FIG. 1. (Color online) The equienergy contours at the satellite points $\left(K_{A}, K_{B}, K_{C}\right)$ and original Dirac point $K$.

where

$$
\begin{aligned}
\Delta & =\frac{\lambda_{R}}{t_{h}}, \quad \varphi_{0}=e^{i a k_{y} / \sqrt{3}}\left[1+2 e^{-(1 / 2) i \sqrt{3} a k_{y}} \cos \left(\frac{a k_{x}}{2}\right)\right], \\
\varphi_{+} & =e^{i a k_{y} / \sqrt{3}}\left[1-2 e^{-(1 / 2) i \sqrt{3} a k_{y}} \sin \left(\frac{a k_{x}}{2}+\frac{\pi}{6}\right)\right], \\
\varphi_{-} & =e^{i a k_{y} / \sqrt{3}}\left[1+2 e^{-(1 / 2) i \sqrt{3} a k_{y}} \sin \left(\frac{a k_{x}}{2}-\frac{\pi}{6}\right)\right] .
\end{aligned}
$$

The eigenvalues of Eq. (6) are given by

$$
E_{\eta_{1}, \eta_{2}}(k)=\eta_{1} t_{h} \sqrt{W+\eta_{2} \sqrt{W^{2}-\left|\varphi_{0}^{2}+\Delta^{2} \varphi_{-} \varphi_{+}\right|^{2}}},
$$

where $W=\left|\varphi_{0}\right|^{2}+\Delta^{2}\left(\frac{\left|\varphi_{+}\right|^{2}+\left|\varphi_{-}\right|^{2}}{2}\right)$ and $\eta_{1}, \eta_{2}= \pm 1$ for the different energy branches. Because the spin-orbit interaction breaks the SU(2) invariance, the twofold degenerate states for electron and hole are lifted respectively. However, for the points like $K, K^{\prime}$ possessing the high symmetry there still exists double essential degeneracy which is at the original Dirac points. Moreover, there are some double accidental degeneracy points which are dependent on the coupling parameter $\Delta$.

In the low-energy limit a quantum system with dispersion relation geometry shaped like a Dirac cone is characterized by the effective Hamiltonian $H=\hbar v_{F} \vec{\sigma} \cdot \vec{k}$, which is a massless two-dimensional (2D) Dirac equation. The Hamiltonian gives the dispersion relation $E(k)=\hbar v_{F} k$. The contribution to the conductivity from a Dirac cone has been discussed in detail in Refs. [36,37]. By direct observation, one can see that equienergy contour at $K_{A}, K_{B}, K_{C}$ is a ellipselike shape as shown in Fig. 1 which at $K_{0}$ is strictly a circle. The difference between the $K_{0}$ and $K_{A(B, C)}$ is of course more fundamental. The Chern number for each gapless point is defined as $C_{K_{i}}=(1 / 2 \pi i) \int_{0}^{2 \pi} d \phi A_{\phi}$, where $A_{\phi}^{\left(K_{i}\right)}$ is the Berry 
phase and $\tan \phi=\delta k_{x} / \delta k_{y}$. For the center gapless point [15] $A_{\phi}^{\left(K_{0}\right)}=-i(1 / 2)$, and $C_{K_{0}}=-1 / 2$. For the three satellite gapless points, $C_{K_{1}}=C_{K_{2}}=C_{K_{3}}=1 / 2$. The nonzero Chern numbers of states at $K_{0}, K_{1}, K_{2}$, and $K_{3}$ manifest that the wave function can acquire a (Berry) phase [42], $\gamma_{K_{i}}=2 \pi C_{K_{i}}$, if the Bloch state of an electron travels a closed circle around these points.

The shape changing has an influence on the validity of the model Hamiltonian $\hat{H}=\hbar v_{F} \vec{\sigma} \cdot \vec{k}=v_{F} \vec{\sigma} \cdot \vec{p}$ where the dispersion relation geometry is not exactly a conic section. Considering the symmetry of the Brillouin zone we pay more attention to the band structure around the point $K_{A}$ where $\left(K_{A}\right)_{y}=0$ for simplicity. To this end, we expand the branch $E_{-1,-1}^{2}\left(k_{x}, k_{y}\right)$ to the second order at the point $K_{A}=\left\{2 \cos ^{-1}\left(\frac{2 \Delta^{2}-1}{2\left(\Delta^{2}+1\right)}\right), 0\right\} \frac{1}{a}$ and obtain

$$
E_{-1,-1}^{2}\left(k_{x}, k_{y}\right) \approx\left(\hbar v_{F}\right)^{2}\left(\zeta_{x}^{2} \delta k_{x}^{2}+\zeta_{y}^{2} \delta k_{y}^{2}\right),
$$

where $\delta k_{x}=k_{x}-K_{A x}, \quad \delta k_{y}=k_{y}-K_{A y}, \quad \zeta_{x}^{2}=\Delta^{2}, \quad \zeta_{y}^{2}=$ $9 \Delta^{2}\left(1-4 \Delta^{2}\right)$, and the relation $\hbar v_{F}=\frac{\sqrt{3}}{2} t_{h} a$ is used. It follows from Eq. (8) that the effective modified dispersion relation is $E_{K_{A}}\left(k_{x}, k_{y}\right)=\hbar v_{F} \sqrt{\left(\zeta_{x} \delta k_{x}\right)^{2}+\left(\zeta_{y} \delta k_{y}\right)^{2}}$ with the constraint $\Delta \neq 0$. Therefore, the Hamiltonian of the effective quantum system characterized by the dispersion geometry in the neighborhood of the Dirac point $K_{A}$ can be phenomenologically written as

$$
\begin{aligned}
\hat{H}_{K_{A}} & =v_{F} \vec{\sigma} \cdot \vec{p}^{\prime}=v_{F}\left(\begin{array}{cc}
0 & p_{-}^{\prime} \\
p_{+}^{\prime} & 0
\end{array}\right) \\
& =v_{F}\left(\begin{array}{cc}
0 & \zeta_{x} p_{x}-i \zeta_{y} p_{y} \\
\zeta_{x} p_{x}+i \zeta_{y} p_{y} & 0
\end{array}\right),
\end{aligned}
$$

where $\quad p_{-}^{\prime}=p_{x}^{\prime}-i p_{y}^{\prime}, p_{+}^{\prime}=p_{x}^{\prime}+i p_{y}^{\prime}, p_{x}^{\prime} \equiv \zeta_{x} \hbar \delta k_{x}=$ $\zeta_{x} p_{x}, p_{y}^{\prime} \equiv \zeta_{y} \hbar \delta k_{y}=\zeta_{y} p_{y} \quad$ with defining $p_{-}^{\prime} p_{+}^{\prime} \equiv p^{\prime 2}=$ $\left(\zeta_{x} p_{x}\right)^{2}+\left(\zeta_{y} p_{y}\right)^{2}=p_{x}^{\prime 2}+p_{y}^{\prime 2}$. When the time-harmonic external field $\vec{A}(t)=\vec{A}_{0} \exp ^{-i \omega t}=\frac{\vec{E}(t)}{i \omega}$ is applied on the system in terms of the principle of minimal coupling the Hamiltonian in the external field is expressed as

$$
\hat{H}_{K_{A}, \text { inter }}=v_{F}\left(\begin{array}{cc}
0 & p_{-}^{\prime}+e A_{-} \\
p_{+}^{\prime}+e A+ & 0
\end{array}\right),
$$

where $A_{-}=\zeta_{x} A_{x}-i \zeta_{y} A_{y}, A_{+}=\zeta_{x} A_{x}+i \zeta_{y} A_{y}$. For simplicity, it is assumed that $A_{y}=E_{y}=0$ and then $A_{-}=A_{+}=$ $\zeta_{x} A_{x}=\frac{\zeta_{x} E_{x}}{i \omega}=\frac{\zeta_{x} E}{i \omega} \exp ^{-i \omega t}$ with $\left|E_{x}\right| \equiv E$. Therefore, we only focus on the specific element $\sigma_{x, x, x, x}^{(3)}$ with the definition $J_{i}^{(3)}=\sigma_{i, l, m, n}^{(3)} E_{l} E_{m} E_{n}$

According to the Floquet theorem, the time-dependent two-component wave functions are expanded as $\psi(p, t)=$ $\sum_{n=0}^{\infty}\left[{ }_{\beta_{n}(p)}^{\alpha_{n}(p)}\right] e^{i n \omega t} e^{-i \varepsilon t / \hbar}$. After substituting it into the Schrödinger equation we get the similar energy-momentum relation $\varepsilon=v_{F} p^{\prime}$. The current excited by the external field $\vec{A}(t)$ is given by

$$
\vec{J}=\frac{e}{4 \pi^{2} \hbar^{2}} \int\left\langle\vec{v}\left(p_{x}, p_{y}\right)\right\rangle N[\varepsilon] d p_{x} d p_{y}
$$

where $\langle\vec{v}(p)\rangle=\psi^{\dagger} \hat{v} \psi$ and $N(\varepsilon)=n_{F}(-\varepsilon)-n_{F}(\varepsilon)$ is the thermal factor [37]. According to the definition of velocity operator $\hat{\vec{v}}=\frac{\partial \hat{H}(p)}{\partial \vec{p}}$, the component of velocity is modified as $\hat{v}_{x}=\frac{\partial \hat{H}(p)}{\partial p_{x}}=\frac{\partial \hat{H}\left(\vec{p}^{\prime}\right)}{\partial p_{x}^{\prime}} \frac{\partial p_{x}^{\prime}}{\partial p_{x}}=\zeta_{x} v_{F} \sigma_{x}=$ $\zeta_{x}{\hat{v^{\prime}}}_{x}, \hat{v}_{y}=\zeta_{y} v_{F} \sigma_{y}=\zeta_{x}{\hat{v^{\prime}}}_{y}$. The integral measure $d p_{x} d p_{y} \rightarrow$ $\frac{d p_{x}^{\prime} d p_{y}^{\prime}}{\left|\zeta_{x} \zeta_{y}\right|}$ in Eq. (11).

Because the domain of integration is over all space the linear transformation between $\vec{p}$ and $\vec{p}^{\prime}$ in Eq. (11) become

$$
\vec{J}=\frac{1}{\zeta_{y}} \frac{e}{4 \pi^{2} \hbar} \int\left\langle\vec{v}^{\prime}\left(p_{x}^{\prime}, p_{y}^{\prime}\right)\right\rangle N\left[\varepsilon\left(p^{\prime}\right)\right] d p_{x}^{\prime} d p_{y}^{\prime} .
$$

Comparing Eq. (12) with the case in Refs. [36,37] and noting $E_{0}^{3} \rightarrow\left(\zeta_{x} E_{0}\right)^{3}$ we get the modified third-order conductivity when the direction of the applied field $\vec{E}(t)=E_{0} e^{i \omega t}$ is along the $x$ axis:

$$
\sigma_{3}^{M}=\frac{\zeta_{x}^{3}}{\zeta_{y}} \sigma_{3}^{D}
$$

where $\sigma_{3}^{D}=\frac{e^{2}}{4 \hbar} \frac{e^{2} E_{0}^{2}}{\hbar^{2} \omega^{4}}\left[N_{3}(\omega) e^{i 3 \omega t}+N_{1}(\omega) e^{i \omega t}\right] v_{F}^{2}$ is the thirdorder conductivity that is contributed by the perfect Dirac cone [36,37] with the Chern number $-1 / 2$. For the points $K_{B}, K_{C}$ it follows from the symmetry of the Brillouin zone that the dispersion relation around them is $E_{K_{B / C}}\left(k_{x}, k_{y}\right)=\hbar v_{F} \sqrt{\left(\zeta_{x} \delta k_{x}^{\prime}\right)^{2}+\left(\zeta_{y} \delta k_{y}^{\prime}\right)^{2}}$, where $\left(\begin{array}{l}\delta k_{x}^{\prime} \\ \delta k_{y}^{\prime}\end{array}\right)=$ $\left(\begin{array}{cc}\cos \theta_{0} & -\sin \theta_{0} \\ \sin \theta_{0} & \cos \theta_{0}\end{array}\right)\left(\begin{array}{c}\delta k_{x} \\ \delta k_{y}\end{array}\right)$ and $\theta_{0}=\mp \frac{\pi}{3}$ for $K_{B}, K_{C}$. By redefining $\left(\begin{array}{l}p_{x}^{\prime} \\ p_{y}^{\prime}\end{array}\right)=\hbar\left(\begin{array}{cc}\cos \theta_{0} & -\sin \theta_{0} \\ \sin \theta_{0} & \cos \theta_{0}\end{array}\right)\left(\begin{array}{cc}\zeta_{x} & 0 \\ 0 & \zeta_{y}\end{array}\right)\left(\begin{array}{l}\delta k_{x} \\ \delta k_{y}\end{array}\right)$, we obtain the same effective Hamiltonian as the one in Eq. (9).

For the essential degeneracy point $K$ where the trigonal warping effect occurs, although the geometry of the dispersion relation can be described by well-defined Dirac cone, the slope of the cone line should be modified under the condition $\Delta \neq 0$. In Fig. 2 the derivative Dirac cones at $K_{A}$ are given with the different $\Delta$ for the case $k_{y}=0\left(k_{x} \neq 0\right)$. It follows from Fig. 2 that when $\Delta \rightarrow 0$ the derivative Dirac cones, which results from the accidental degeneracy, approach the point $K$ and the height of the effective Dirac cones also descends and vanishes eventually. Although the slope of the original Dirac cone is influenced by the nonzero RSOC the circular shape of the equifrequency contour is invariant. Because of the singularity of the derivative of $E_{ \pm 1,-1}\left(k_{x}, 0\right)$ at $K$ (shown in Fig. 2) we

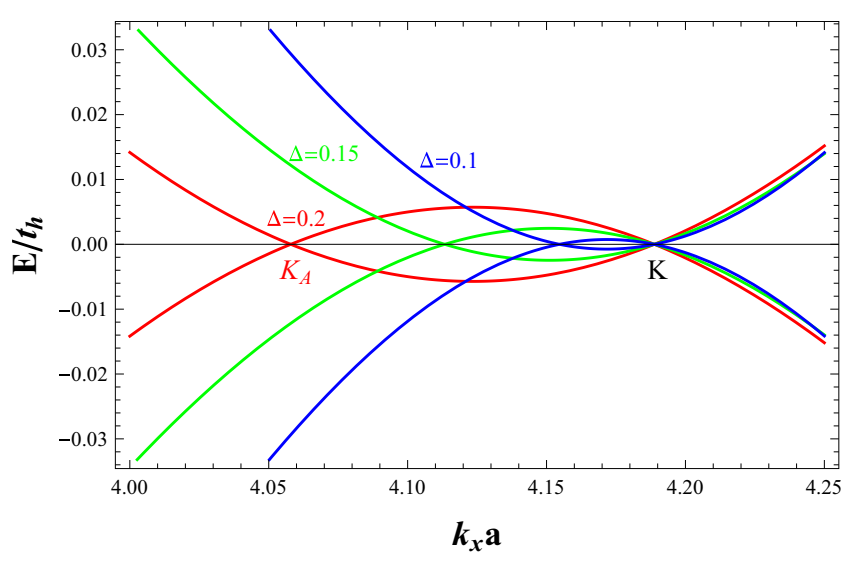

FIG. 2. (Color online) The Dirac cones at the satellite point $K_{A}$ for different coupling strength $\Delta=0.2,0.15,0.1$. 


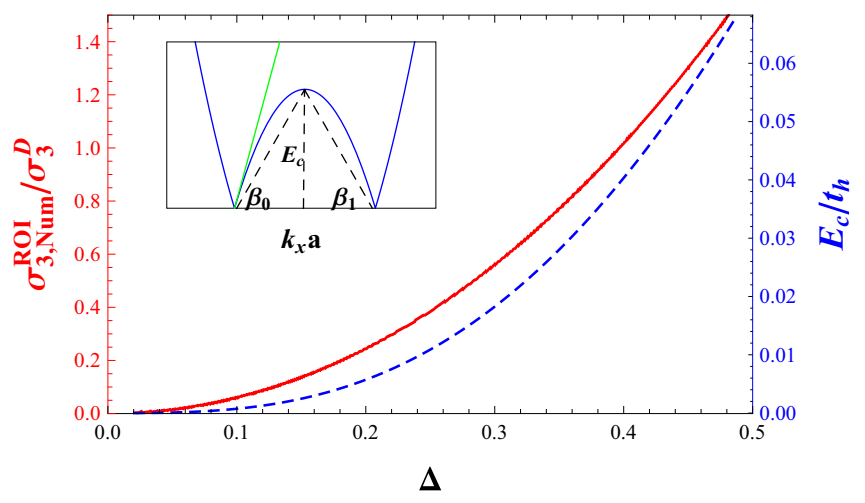

FIG. 3. (Color online) Red solid line: Third-order conductivity with RSOC vs the coupling coefficient $\Delta$ for the fixed terahertz frequency. Blue dashed line: The maximal energy of the incoming photon for the validity of the linear model.

take a one-sided limit to get the modified slope,

$$
\lim _{k \rightarrow K^{-}} \frac{\partial E_{ \pm 1,-1}\left(k_{x}, 0\right)}{\partial k_{x}}=\mp \frac{\sqrt{3}}{2} \Delta t_{h} a=\mp \hbar v_{F} \Delta .
$$

This slope in Eq. (14) is hinted at by the slope of the green line tangent to the cone at $K$ in the inset of Fig. 3. The effective Hamiltonian at $K$ becomes $H_{K}=v_{F}^{\prime}\left(\begin{array}{cc}0 & p_{-} \\ p_{+} & 0\end{array}\right)$ with $v_{F}^{\prime}=v_{F} \Delta$ and the contribution to the conductivity from the cone at $K$ reads

$$
\sigma_{3, K}^{M}=\Delta^{2} \sigma_{3}^{D},
$$

which is reduced into the case that $\zeta_{y}=\zeta_{x}=\Delta$ in Eq. (13).

For typical RSOC parameters, $\Delta<1$ and the height of the cones are much smaller than the other two energy branches $E_{ \pm 1,-1} \ll E_{ \pm 1,+1} . E_{+1,+1}-E_{-1,+1}=\delta E$ fall into the terahertz regime. For photon energy lower than the height of the cones, the third-order nonlinear conductivity with RSOC is expressed as

$$
\sigma_{3}^{\mathrm{RSOC}}=6\left(\frac{3 \zeta_{x}^{3}}{\zeta_{y}}+\Delta^{2}\right) \sigma_{3}^{D}
$$

Before we go on to analyze the feature of Eq. (16), investigating the conductivity numerically is useful to shed light on the physical mechanism of the nonlinear conductivity with RSOC. To this end we ignore the effect of the deformation for the moment and approximate the slope of the line that connects the vault and the Dirac points as the effective slope of the cones, as shown (dashed line) in the inset of Fig. 3. Denoting the angle between the dashed lines and the $k_{x}$ axis as $\beta_{0}(K), \beta_{1}\left(K_{A}\right)$ respectively and noting that the dimensionless slope of the standard Dirac cone $\left(E=v_{F} \hbar k\right)$ is $\frac{\sqrt{3}}{2}$, the third-order nonlinear conductivity is numerically obtained:

$$
\sigma_{3, \text { Num }}^{\text {RSOC }}=8\left(\tan ^{2} \beta_{0}+3 \tan ^{2} \beta_{1}\right) \sigma_{3}^{D} .
$$

The calculated nonlinear conductivity is shown in Fig. 3 (red solid line). It is easily found that the nonlinear conductivity with ROI is obviously enhanced for sufficiently large $\Delta$. Moreover, the enhancement is independent of frequency, which is the manifestation of topological change of the energy

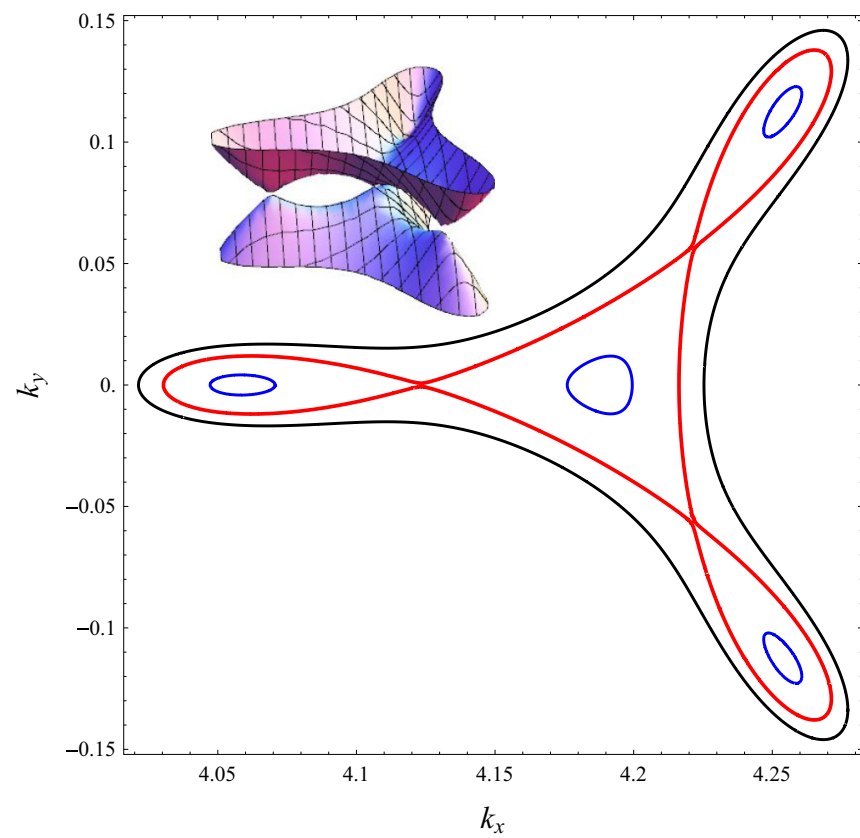

FIG. 4. (Color online) Equienergy contour at $E_{1,-1}(\vec{k}) \times$ $10^{3} / t_{h}=8.0$ (black), 5.73 (red), 2.0 (blue) with $\Delta=0.2$.

band from the RSOC. It is mainly due to the increase of the effective Fermi velocity (the slope) of the cones with the enhancement of coupling strength $\Delta$. It is notable that there is a restriction that the photon energy must be always lower than the cone height. The strong enhancement shown above is protected by the topology of the band structure in the presence of RSOC. In other words, it is protected by the trigonal warping at low energy. For each fixed $\Delta$ there exists a critical value of energy $E_{c}(\Delta)$ at which the equienergy contour has three self-intersection points shown with a red line in Fig. 4 and this energy value $E_{c}$ is defined as the height of the Dirac cone. When the energy of the incoming photon is larger than $E_{c}$ the equienergy contour contributing to the conductivity by the resonance transition becomes a simple closed curve shown in Fig. 4 (black line) which is not topologically equivalent to the one that our model holds for where the equienergy contour is composed of three discrete closed curves shown with a blue line in Fig. 4.

The comparison of nonlinear conductivity from Eq. (17) (red line) and from Eq. (16) (blue line) is shown in Fig. 5. It can be found that the results with the different methods are consistent with each other qualitatively. Moreover, for the fixed $\Delta$ the conductivity given by Eq. (16) is larger than that given by (17) where the effect of the deformation is ignored. It also indicates that the effect of the deformation of the cone is important for the enhancement of the conductivity.

To determine the value of $E_{c}$ we chose the derivative of the curve $\frac{\partial\left(E_{1,-1} \mid k_{y}=0\right)}{\partial k_{x}}=0$ for simplicity due to the symmetry. The numerical solution to the relevant transcendental equation is shown (blue dashed line) in Fig. 3 and it follows from the curve that $E_{c}$ decreases monotonously with the decay of $\Delta$. When the parameter $\Delta$ changes continuously there are two classes of topologically inequivalent equienergy contours (See Fig. 4) and our model only holds for one of the two classes 


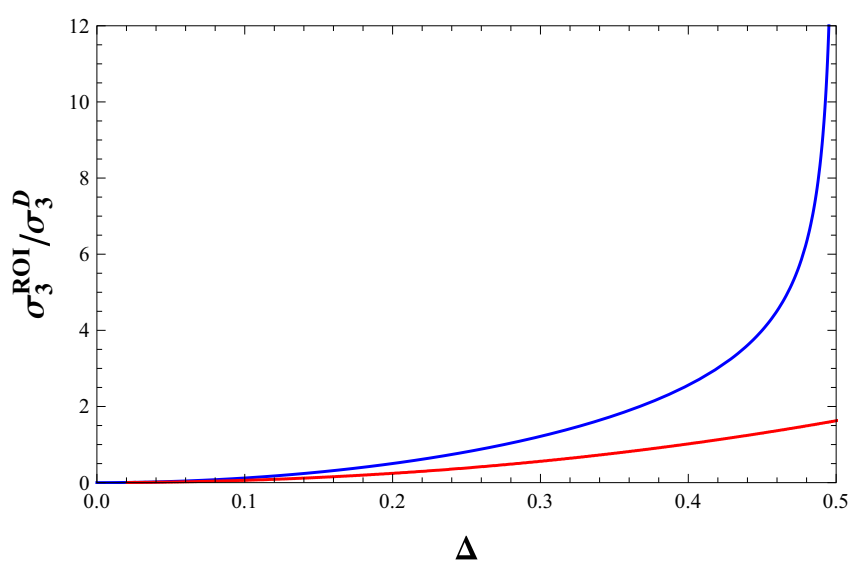

FIG. 5. (Color online) Third-order conductivity with RSOC vs the coupling coefficient $\Delta$ for Eq. (17) (red line) and Eq. (16) (blue line).

(blue contours). Therefore, the energy of the incoming photon $\left(\hbar \omega_{\text {in }}\right)$ and $\Delta$ are restricted because of the validity of our model and the constraint can be written explicitly as $\hbar \omega_{\text {in }}<E_{c}(\Delta)$.

\section{CONCLUSIONS}

In summary we have shown that the third-order nonlinear conductivity of graphene can be distinctively enhanced in the presence of RSOC in the regime where the frequency is below the cone height defined by the spin-orbit interaction, $\hbar \omega_{\text {in }}<E_{c}(\Delta)$. The enhancement originates from topologically inequivalent change of the energy bands around the original Dirac point and the shape changing of the derivative Dirac cones. The shape changing of the cones leads to a rapid increase of the density of states at low energies. While the topological change of the band structure results in a six times enhancement of the linear conductivity, it has a much stronger effect on the nonlinear conductivity. The result presented here can be readily applied to the nonlinear conductivity of a bilayer graphene.

\section{ACKNOWLEDGMENTS}

This work was supported by the 973 Program of China (Grant No. 2014CB339803), the 863 Program of China (Project No. 2011AA010205), the National Natural Science Foundation of China (Grants No. 61131006, No. 61321492, and No. 11174309), the Major National Development Project of Scientific Instrument and Equipment (Grant No. 2011YQ150021), the National Science and Technology Major Project (Grant No. 2011ZX02707), the Major Project (Project No. YYYJ-1123-1), the International Collaboration and Innovation Program on High Mobility Materials Engineering of the Chinese Academy of Sciences, and the Australian Research Council Grant through a Discovery Grant (Grant No. DP140101501).
[1] K. S. Novoselov, A. K. Geim, S. V. Morozov, D. Jiang, Y. Zhang, S. V. Dubonos, I. V. Grigorieva, and A. A. Firsov, Science 306, 666 (2004).

[2] Y. Zhang, J. P. Small, Michael E. S. Amori, and P. Kim, Phys. Rev. Lett. 94, 176803 (2005).

[3] C. Berger, Z. Song, T. Li, X. Li, A. Y. Ogbazghi, R. Feng, Z. Dai, A. N. Marchenkov, E. H. Conrad, P. N. First, and W. A. de Heer, J. Phys. Chem. B 108, 19912 (2004).

[4] Y. Zhang, Y. W. Tan, H. L. Stormer, and P. Kim, Nature (London) 438, 201 (2005).

[5] K. S. Novoselov, A. K. Geim, S. V. Morozov, D. Jiang, M. I. Katsnelson, I. V. Grigorieva, S. V. Dubonos, and A. A. Firsov, Nature (London) 438, 197 (2005).

[6] M. I. Katsnelson, K. S. Novoselov, and A. K. Geim, Nat. Phys. 2, 620 (2006).

[7] A. K. Geim and K. S. Novoselov, Nat. Mater. 6, 183 (2007).

[8] R. Saito, G. Dresselhaus, and M. S. Dresselhaus, Phys. Rev. B 61, 2981 (2000).

[9] P. Rakyta, A. Kormányos, and J. Cserti, Phys. Rev. B 82, 113405 (2010).

[10] J. Cserti, A. Csordás, and G. Dávid, Phys. Rev. Lett. 99, 066802 (2007).

[11] C. L. Kane and E. J. Mele, Phys. Rev. Lett. 95, 226801 (2005).

[12] A. Cortijo, A. G. Grushin, and M. A. H. Vozmediano, Phys. Rev. B 82, 195438 (2010).

[13] Yu. S. Dedkov, M. Fonin, U. Rüdiger, and C. Laubschat, Phys. Rev. Lett. 100, 107602 (2008).
[14] P. Ingenhoven, J. Z. Bernád, U. Zülicke, and R. Egger, Phys. Rev. B 81, 035421 (2010).

[15] W. Wang, C. Zhang, and Z. Ma, J. Phys.: Condens. Matter 24, 035303 (2012).

[16] F. Guinea, New J. Phys. 12, 083063 (2010).

[17] R. van Gelderen and C. M. Smith, Phys. Rev. B 81, 125435 (2010).

[18] E. McCann and M. Koshino, Phys. Rev. B 81, 241409(R) (2010).

[19] H. Min, J. E. Hill, N. A. Sinitsyn, B. R. Sahu, L. Kleinman, and A. H. MacDonald, Phys. Rev. B 74, 165310 (2006).

[20] A. H. Castro Neto and F. Guinea, Phys. Rev. Lett. 103, 026804 (2009).

[21] M. Zarea and N. Sandler, Phys. Rev. B 79, 165442 (2009).

[22] T. Fukui and Y. Hatsugai, Phys. Rev. B 75, 121403(R) (2007).

[23] M. Gmitra, S. Konschuh, C. Ertler, C. Ambrosch-Draxl, and J. Fabian, Phys. Rev. B 80, 235431 (2009).

[24] E. McCann, D. S. L. Abergel, and V. I. Fal'ko, Solid State Commun. 143, 110 (2007).

[25] G. P. Mikitik and Yu. V. Sharlai, Phys. Rev. B 77, 113407 (2008).

[26] M. Koshino, New J. Phys. 11, 095010 (2009).

[27] M. Koshino and E. McCann, Phys. Rev. B 80, 165409 (2009).

[28] R. R. Nair, P. Blake, A. N. Grigorenko, K. S. Novoselov, T. J. Booth, T. Stauber, N. M. R. Peres, and A. K. Geim, Science 320, 1308 (2008).

[29] A. R. Wright and C. Zhang, Phys. Rev. B 81, 165413 (2010).

[30] V. Ryzhiia, M. Ryzhii, and T. Otsuji, J. Appl. Phys. 101, 083114 (2007). 
[31] L. A. Falkovsky and A. A. Varlamov, Eur. Phys. J. B 56, 281 (2007).

[32] V. P. Gusynin, S. G. Sharapov, and J. P. Carbotte, Phys. Rev. Lett. 96, 256802 (2006).

[33] A. R. Wright, J. C. Cao, and C. Zhang, Phys. Rev. Lett. 103, 207401 (2009).

[34] J. Liu, A. R. Wright, C. Zhang, and Z. Ma, Appl. Phys. Lett. 93, 041106 (2008).

[35] Y. Lemonik, I. L. Aleiner, C. Toke, and V. I. Fal'ko, Phys. Rev. B 82, 201408(R) (2010).
[36] A. R. Wright, X. G. Xu, J. C. Cao, and C. Zhang, Appl. Phys. Lett. 95, 072101 (2009).

[37] X. G. Xu, S. Sultan, C. Zhang, and J. C. Cao, Appl. Phys. Lett. 97, 011907 (2010).

[38] C. Zhang, L. Chen, and Z. Ma, Phys. Rev. B 77, 241402 (2008).

[39] S. A. Mikhailov, Microelectron. J. 40, 712 (2009).

[40] G. W. Semenoff, Phys. Rev. Lett. 53, 2449 (1984).

[41] A. De Martino, R. Egger, K. Hallberg, and C. A. Balseiro, Phys. Rev. Lett. 88, 206402 (2002).

[42] M. V. Berry, Proc. R. Soc. London Ser. A 392, 45 (1984). 